\title{
Note: Slowing-down of diffusion-controlled reactions in dense liquid matter
}

Cite as: J. Chem. Phys. 138, 186101 (2013); https://doi.org/10.1063/1.4804417

Submitted: 26 February 2013 . Accepted: 26 April 2013 . Published Online: 10 May 2013

\section{A. Zaccone}
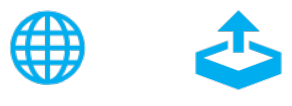

\section{ARTICLES YOU MAY BE INTERESTED IN}

Concentration effects on the rates of irreversible diffusion-influenced reactions

The Journal of Chemical Physics 141, 084101 (2014); https://doi.org/10.1063/1.4893340

A solution of the Debye-Smoluchowski equation for the rate of reaction of ions in dilute solution

The Journal of Chemical Physics 70, 4001 (1979); https://doi.org/10.1063/1.438020

Phase diagrams of Lennard-Jones fluids

The Journal of Chemical Physics 96, 8639 (1992); https://doi.org/10.1063/1.462271

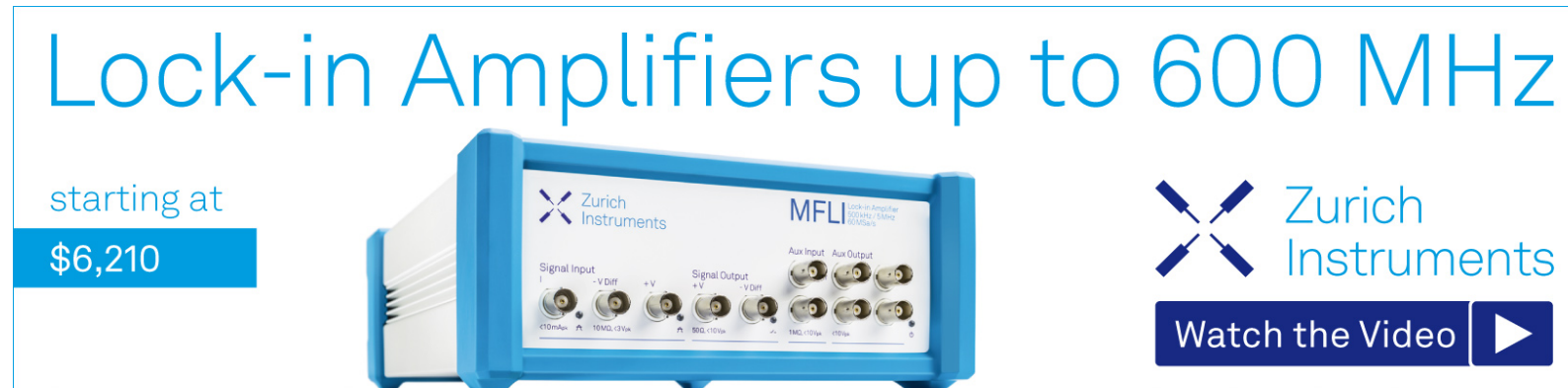




\title{
Note: Slowing-down of diffusion-controlled reactions in dense liquid matter
}

\author{
A. Zaccone \\ Cavendish Laboratory, University of Cambridge, JJ Thomson Avenue, Cambridge CB3 OHE, United Kingdom
}

(Received 26 February 2013; accepted 26 April 2013; published online 10 May 2013)

[http://dx.doi.org/10.1063/1.4804417]

Let us consider the following classical problem of diffusion-controlled reaction kinetics: ${ }^{1,2}$

$$
A+B \rightarrow B,
$$

where $B$ denotes dilute, mobile spherical traps which irreversibly transform the reactant molecules $A$ into the reaction products, upon collision. In the spherical frame centered on the trap, the density distribution of $A$ is denoted by $\rho(r)$. Furthermore, the $A$ molecules are spherical and inert with each other apart from excluded-volume (hard-sphere) repulsion. Denoting with $R_{S}$ the radius of $B$ and with $R_{p}$ the radius of $A$, we focus on the case where $B$ is a sphere of the same size as $A$ and the reaction radius is thus equal to the collision diameter $R=R_{S} / 2=R_{p} / 2$. Furthermore, we neglect hydrodynamic interactions, ${ }^{3}$ which are neglected also in the simulations that we compare our results with. We are interested in the steadystate reaction rate $\kappa$ under the effect of many-particle correlations between $A$ molecules, i.e., at high concentrations of $A$ in the system. This quantity is important in a number of problems, from biophysical receptor-ligand reactions in crowded biological environments, ${ }^{4}$ to exciton recombination in semiconductors, colloidal aggregation, ${ }^{5}$ and matter-antimatter segregation in cosmology. ${ }^{6}$

The problem has been studied recently using event driven Brownian dynamics simulations ${ }^{7}$ over a broad range of packing fraction $\phi=(4 \pi / 3) R_{p}^{3} \rho_{\infty}$ and for several ratios of the trap to reactant radii, $R_{S} / R_{p}$. For moderate values of $\phi$ up to $\phi=0.2$, the rate increases upon increasing $\phi$ due to enhanced collective diffusion, while the density profile around the trap remains monotonic and gradually decreases from the bulk value to zero at the trap. At high liquid density $\phi \gtrsim 0.4$, the rate is slowed down. The slowing down was thought to be due to the build-up of many-body excluded-volume correlations between $A$ molecules around the trap, which, at high density, cannot be removed by the local depletion effect created by annihilation of $A$ molecules at the trap.,

In Ref. 7, it was found that the simulation data for the rate can be well represented, omitting numerical prefactors, by the following expression:

$$
\kappa \simeq \kappa_{S} \frac{\beta \Pi\left(\rho_{\infty}\right)}{\rho_{\infty}} e^{-\left[\beta \Pi\left(\rho_{\Delta}\right) / \rho_{\Delta}\right]+1},
$$

where $\kappa_{S}=4 \pi D_{0} R \rho_{\infty}$ is the Smoluchowski diffusion-limited rate valid in the limit $\rho \rightarrow 0$, and $\rho_{\Delta} \equiv \rho\left(r_{\Delta}\right)$ is evaluated at the first coordination shell in the density pair-distribution function $\rho(r)$ due to mutual excluded volume of $A$ molecules around the trap. The exponential factor has been interpreted in Refs. 7 and 8 as an effective Arrhenius factor with a thermally-activated energy barrier. The latter is connected with the local build-up of osmotic pressure which a generic $A$ molecule feels due to the first coordination shell of other $A$ molecules crowding around $B$. Although this concept is intuitively appealing, there is no formal, first-principles derivation of Eq. (2) available.

The equilibrium structure of substrate fluid (the $A$ particles), is perturbed near the trap $B$ due to the removal (depletion) of the $A$ particles upon collision with the trap. Under dilute conditions such that the probability density distribution of $A$ is uniform at all length scales, the profile of the pair distribution function of $A$ particles around $B$ is given by $\rho(r)$ $=\rho_{\infty}[1-(R / r)]$, which is the Smoluchowski solution to the steady two-body diffusion describing transport of the $A$ particle subject to the absorbing boundary condition at contact with $B$. At high density of $A$ particles, this profile is further perturbed by the liquid-like short-range structure induced by many-body hard-core repulsions. ${ }^{7}$

The transport of one $A$ particle from the bulk to the trap $B$ occurs through the inhomogeneous local density profile due to the many-particle correlations (liquid structure). In the presence of an inhomogeneous density profile, a Brownian particle is subjected to a force which acts as to spread the density profile. Recall that in a system of Brownian particles, $\Pi$ is the pressure exerted by the system itself. Then $-\nabla \Pi$ represents the force per unit volume acting on the system. ${ }^{9}$ If $\rho$ is the probability density of particles, it follows that the force acting on average upon a single particle is given by $-\rho^{-1} \nabla \Pi$. Hence, this force is zero whenever the system is spatially homogeneous, as it ought to be, e.g., for diluted suspensions. If, however, the distribution of the particles is not homogeneous in space, this force is written as $F=-\frac{1}{\rho(\mathbf{r})} \nabla \Pi(\rho(\mathbf{r}))$. It is important to realize that this is a non-conservative forcefield, as it is connected with an irreversible diffusive flux, and therefore it differs from the usual potential of mean-force of equilibrium physics. ${ }^{10}$

The reaction kinetics is governed by the diffusive FokkerPlanck equation:

$$
\frac{\partial \rho}{\partial t}=D_{c} \nabla \cdot(\nabla \rho-\beta F \rho)
$$

In this problem, many-body correlation effects arise on two levels. One level is that of collective diffusion contained in $D_{c}=D_{0}(\partial \Pi / \partial \rho)$, reflecting the effect of many-particle collisions on transport in a dense system. ${ }^{9}$ The second level is represented by the inhomogeneous static liquid structure which gives the spatial modulation of $\rho(r)$, as the solution of 
integral equations, such as, e.g., the Hypernetted Chain Equation (HNC). The two levels are nonlinearly coupled through $D_{c}(\rho)$ and the full formalization of the problem involves the coupling of the HNC and the Fokker-Planck equations, a formidable task even for numerics.

The first approximation is to assume a superimposed liquid structure $\rho_{S}(r)$ as given, e.g., by the HNC or from experiments, assumed independent of the two-particle density function $\rho(r)$ of the two annihilating particles, to effectively decouple the structure from the transport. The second approximation is to reformulate the many-body transport problem as a homogeneous collective diffusion problem in the superimposed field of the nonequilibrium, inhomogeneous mean-force $F\left(\rho_{S}(r)\right)$ introduced above. This amounts to linearizing the transport problem, while keeping the nonequilibrium effect of the diffusion through an inhomogeneous structure via $F\left(\rho_{S}(r)\right)$. This further reduction step leads to replacing $D_{c}$ in Eq. (3) with $D_{c, \infty}$.

The steady-state solution of the Fokker-Planck equation for the flux is $\kappa=4 \pi r^{2} D_{c}(\nabla \rho-\beta F \rho)$. Upon integrating with the condition $\rho=\rho_{\infty}$ at $r=\infty$, we obtain the steady-state solution as

$$
\rho=\rho_{\infty} e^{\int d r \beta F}+\frac{\kappa e^{\int d r \beta F}}{4 \pi D_{c, \infty}} \int_{\infty}^{r} \frac{1}{r^{2}} e^{-\int d r \beta F} d r
$$

The integrated annihilation rate is given using the absorbing boundary condition at the trap, $\rho=0$ at $r=R$, as

$$
\kappa=\frac{8 \pi D_{c, \infty} \rho_{\infty}}{\int_{R}^{\infty} r^{-2} e^{-\int \beta F d r} d r} .
$$

Let us consider the indefinite integral

$$
-\int \beta F d r=\beta \int \frac{1}{\rho} \frac{d \Pi}{d r} d r=\beta \int \frac{1}{\rho} d \Pi .
$$

Practically, this integral is non-zero only about the first coordination shell where the inhomogeneity is the strongest. $\Pi$ is a series expansion of $\rho$ with positive integer powers $1, \ldots$, $N$, and we recall that a high-density liquid equation of state such as the Hall equation of state ${ }^{11}$ can be reduced to this form. Upon inverting the relation, the dependence of $\rho$ on $\Pi$ is controlled by $\sim \Pi^{1 / N}$, where $N$ is the largest power exponent in the series. For example, with $N=7$, the dependence goes as $\sim \Pi^{1 / 7}$. Since this is a very slowly varying function of $\Pi$ in the region which contributes to the integral, we can write

$$
-\int \beta F d r \simeq \frac{1}{\rho} \beta \Pi+C .
$$

The integration constant $C$, has to be such that the structural mean-force $F$ is zero for a homogeneous and ideal system, hence $C=-1$. With these identifications, the rate becomes

$$
\kappa \simeq 4 \pi D_{c, \infty} \rho_{\infty}\left[R \int_{R}^{\infty} r^{-2} e^{(\beta \Pi / \rho)-1} d r\right]^{-1} .
$$

The integral is dominated by the region in $r$-space where $\beta \Pi / \rho>1$. Since $\Pi$ is a monotonically increasing polyno- mial function of $\rho(r)$, to a peak in $\rho(r)$ there corresponds an even sharper peak in $\Pi(r)$. At high density, the spatial behavior of $\rho(r)$ is the same as the radial distribution function, with the largest peak corresponding to the first coordination shell of $A$ particles. The presence of such a peak around the trap has been confirmed by simulations also for the annihilation (absorption) boundary condition at high packing of $A$ particles. ${ }^{7}$ The exponential evaluated at the peak represents the dominant contribution to the integral. In the simplest approximation, we schematize the peak as a square barrier of height $\beta \Pi\left(\rho_{\Delta}\right) / \rho_{\Delta}-1$, where $\rho_{\Delta}$ is evaluated at $r_{\Delta}$ which is the coordinate of the peak, that is, the position of the first coordination shell along $r$. Hence, upon recalling the form of the Smoluchowski rate valid in the ideal limit, $\kappa_{S}=4 \pi D_{0} R \rho_{\infty}$, we arrive at the following result:

$$
\kappa \simeq \kappa_{S}\left(\frac{d \beta \Pi}{d \rho}\right)_{\rho_{\infty}} e^{-\left[\beta \Pi\left(\rho_{\Delta}\right) / \rho_{\Delta}\right]+1} .
$$

This result, obtained using the nonequilibrium mean-force $F$ accounting for the transport through the inhomogeneous short-range structure of dense liquids, can be compared with Eq. (2) that was obtained by fitting simulation data. ${ }^{7}$ The two equations differ only in the prefactor, where in Eq. (2), we have $\beta \Pi\left(\rho_{\infty}\right) / \rho_{\infty}$ instead of $(d \beta \Pi / d \rho)_{\rho_{\infty}}$. It is important to note that the two prefactors have the same dependence on $\rho_{\infty}$, as could be appreciated by writing $\Pi(\rho)$ as a virial expansion. This indicates that our analytical solution is qualitatively correct overall, and the approximations proposed here may affect only the numerical prefactor. This approximation works, at least qualitatively, possibly because the neglected $\rho$ dependence of $D_{c}$, is by definition less nonlinear in $\rho$ than the dependence of $\Pi$ which survives through the approximation and controls the final outcome.

We have shown that the diffusion-controlled rate of annihilation reactions in dense hard-sphere liquids with dilute mobile traps can be reformulated as a two-body Smoluchowski problem in a locally inhomogeneous, crowding mean-force field. This compact, effective two-body formulation of the many-body problem can be used in other reaction kinetics problems in dense phases, in alternative to or in combination with more rigorous, but more involved, fieldtheoretic approaches. ${ }^{12}$

${ }^{1}$ W. Ebeling and I. M. Sokolov, Statistical Thermodynamics and Stochastic Theory of Nonequilibrium Systems (World Scientific, Singapore, 2005).

${ }^{2}$ A. A. Ovchinnikov and Ya. B. Zel'dovich, Chem. Phys. 28, 215 (1978).

${ }^{3}$ J. T. Padding and A. A. Louis, Phys. Rev. E 74, 031402 (2006).

${ }^{4}$ J. Dzubiella and J. A. McCammon, J. Chem. Phys. 122, 184902 (2005).

${ }^{5}$ A. Zaccone et al., J. Phys. Chem. B 112, 6793 (2008).

${ }^{6}$ D. Toussaint and F. Wilczek, J. Chem. Phys. 78, 2642 (1983).

${ }^{7}$ N. Dorsaz et al., Phys. Rev. Lett. 105, 120601 (2010).

${ }^{8}$ A. Zaccone et al., J. Phys. Chem. B 115, 7383 (2011).

${ }^{9} \mathrm{~J}$. Dhont, An Introduction to Dynamics of Colloids (Elsevier, Amsterdam, 1996).

${ }^{10}$ A. Zaccone and E. M. Terentjev, Phys. Rev. E 85, 061202 (2012).

${ }^{11}$ K. R. Hall, J. Chem. Phys. 57, 2252 (1972).

${ }^{12}$ D. C. Mattis and M. L. Glasser, Rev. Mod. Phys. 70, 979 (1998). 